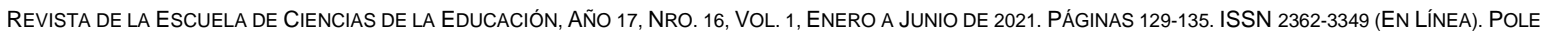
DANCE EN ESTUDIANTES DE LA UNIVERSIDAD AUSTRAL DE CHILE Y SU RELACIÓN CON LA AUTOIMÁGEN: REPERCUSIONES EN LA EDUCACIÓN SUPERIOR. IGNACIA CAROLINA OSORIO NAVARRO. LORETO DANIELA LIBUY CASTRO.

\title{
POLE DANCE EN ESTUDIANTES DE LA UNIVERSIDAD AUSTRAL DE CHILE Y SU RELACIÓN CON LA AUTOIMÁGEN: REPERCUSIONES EN LA EDUCACIÓN SUPERIOR
}

\author{
Ignacia Carolina Osorio Navarro* \\ Escuela México, Valdivia \\ ignaciacarolinaosorionavarro@gmail.com \\ Loreto Daniela Libuy Castro** \\ Universidad Austral de Chile, Chile \\ loreto.libuy@uach.cl
}

Recibido: 11/08/2020 Aceptado: 6/02/2021

\section{Resumen}

El siguiente artículo presenta los resultados obtenidos en una investigación realizada el año 2019 a estudiantes de la Universidad Austral de Chile que practican Pole Dance regularmente, como trabajo de tesis para optar al título de profesora de educación física. Es un estudio cualitativo, donde se analizan los hallazgos a través de tres instrumentos de recolección de datos (encuesta tipo Likert, grupo de discusión y entrevista semiestructurada), los que demuestran que la práctica de este deporte genera repercusiones positivas en la autoimagen de las participantes. Además, se deduce que este deporte se relaciona positivamente con responsabilidades académicas y relaciones sociales dentro de la formación profesional en la educación superior.

Palabras clave: Pole Dance - Autoimagen - Educación superior - Estudiantes.

\footnotetext{
* Licenciada en Educación, Profesora de Educación Física, Deportes y Recreación, Bachiller en Humanidades y Ciencias Sociales (Universidad Austral de Chile). Actualmente encargada de los talleres de danza y gimnasia rítmica en Escuela México, Valdivia; y entrenadora de gimnasia rítmica en Club Cisnes Los Ríos. Estudiante en proceso de obtener la certificación intermedia para instructora de Pole Dance.

* Magister en Motricidad Humana (Universidad Autónoma de Chile), Licenciada en Educación, Profesora de Educación Física (Universidad Católica del Maule), Profesora de Biodanza (Internacional Biocentric Foundation), Educadora Biocéntrica (Universidad Biocéntrica, Fortaleza-Brasil), Diplomada en Psicomotricidad (Universidad Católica del Maule). Actualmente docente del Instituto de Ciencias de la Educación de la Universidad Austral de Chile, Valdivia, y estudiante de Doctorado en Educación en la Universidad Católica del Maule, Chile.
} 


\title{
POLE DANCE AND ITS RELATION WITH UNIVERSIDAD AUSTRAL DE CHILE STUDENTS' SELF IMAGE: REPERCUSSIONS IN HIGHER EDUCATION
}

\begin{abstract}
The following article presents the results obtained in an investigation carried out in 2019 to students of the Austral University of Chile who practice Pole Dance regularly, as thesis work to qualify for the title of physical education teacher. It is a qualitative study, where the findings of three data collection instruments are analyzed (Likert-type survey, discussion group and semi-structured interview), which show that the practice of this sport generates positive repercussions on the self-image of the participants. In addition, it is deduced that this sport is positively related to academic responsibilities and social relations within professional training in higher education.
\end{abstract}

Keywords: Pole Dance - Self-image - Higher education - Students.

\section{Introducción}

La investigación fue realizada el año 2019 en mujeres universitarias que practican Pole Dance regularmente. La hipótesis sostenida es que este deporte puede generar repercusiones positivas en la autoimagen de las estudiantes de la Universidad Austral de Chile de la ciudad de Valdivia, lo que añadiría mejoras en su convivencia social y académica del ambiente universitario.

La metodología de investigación es cualitativa exploratoria, el paradigma es interpretativo y su base teórica se sostiene en autores expertos en educación y educación física, añadiendo una entrevista realizada a una instructora de Pole Dance especializada en el desarrollo de este deporte en Valdivia, para una mayor base teórica que colabora en el análisis de resultados de la investigación.

En términos organizativos, se explica en primera instancia la relación entre Pole Dance y autoimagen. Más adelante, se menciona la metodología de investigación utilizada para responder a los objetivos y problemas de estudio. De este modo, se visualizan los resultados extraídos del análisis de información obtenido por los relatos de las participantes, y se finaliza exponiendo las conclusiones.

\section{Pole Dance y Autoimagen}

El Pole Dance es una disciplina que trabaja la musculatura del cuerpo mientras se realizan destrezas en una barra de acero vertical, que a lo largo de los años se ha convertido en una actividad cada vez más accesible para la comunidad debido a su alza de adeptos. Milena (2014) menciona que el uso de poca ropa mejora el dominio en el tubo, ayudando a la fricción, por lo que es importante trabajar la mayor cantidad de agarres de la piel. Este deporte genera beneficios, sobre todo en aspectos musculares. Morales (2013, p. 37) informa que el Pole Dance "es una forma reconocida de ejercicio y puede ser utilizado como gimnasia aeróbica y acrobática, no requiere una estructura física determinada ni cualidades deportivas extraordinarias". El trabajo en barras responde a las necesidades de los deportistas que realizan trabajos isométricos, sin embargo, también utiliza ejercicios de trabajo concéntrico y excéntrico. Frente a esta situación, Safatle (2016) hace alusión a que el Pole Dance como técnica del desarrollo del cuerpo es excelente, relacionándose con los aspectos mencionados anteriormente.

En la actualidad, existen dos centros que imparten esta disciplina dentro de Valdivia. Se presume que más de la mitad de las participantes que practican Pole Dance son estudiantes mujeres de la Universidad Austral de Chile, con un total de noventa y dos inscritas. Este deporte trabaja intensamente el control corporal, en el proceso, los aprendices dimensionan sus capacidades físicas y psicológicas para realizarlo, adquiriendo confianza como factor fundamental para la disciplina, puesto que varios de los ejercicios necesitan de este aspecto, relacionándose con la manera en que los participantes se vinculan en su ámbito educacional.

El concepto de autoimagen posee diversas interpretaciones. Dentro de los autores que esclarecen el concepto, aparece Valencia (2019) manifestando que la imagen propia se forma a través de las conclusiones que tiene el resto sobre nosotros. Es indispensable que las afirmaciones que se le entreguen al individuo le beneficien de manera fructífera y no busquen hacerlo pensar que no posee las capacidades pertinentes para su crecimiento personal. Cifuentes (2016) aclara que "el adolescente tiene que tener una perspectiva clara y objetiva de su imagen, para determinarse a sí mismo, como la representación ante las demás personas" (Cifuentes, 2016, p. 8). La autoimagen se construye a través de las vivencias de cada persona, por ende, si un sujeto realiza actividades con las cuales siente agrado, puede evolucionar de forma positiva. 


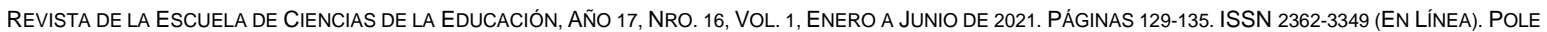
DANCE EN ESTUDIANTES DE LA UNIVERSIDAD AUSTRAL DE CHILE Y SU RELACIÓN CON LA AUTOIMÁGEN: REPERCUSIONES EN LA EDUCACIÓN SUPERIOR. IGNACIA CAROLINA OSORIO NAVARRO. LORETO DANIELA LIBUY CASTRO.

Es recomendable que los estudiantes universitarios realicen alguna actividad que les resulte gratificante, permitiendo que aspectos como el estrés académico se canalicen en la práctica y que los momentos de ocio colaboren con sus responsabilidades universitarias (Rodríguez, 2015). Estas actividades, que los estudiantes eligen en relación a sus preferencias, pueden aumentar la confianza en sí mismos, pues se practica una actividad que les motiva para continuar con su aprendizaje voluntario (Torres, 2012). En el caso del Pole Dance sucede un efecto muy similar a lo mencionado por Torres, pero que a la vez contiene un diferenciador potente en relación a la autoestima de sus participantes, refiriéndonos a la piel y su agarre en el tubo.

Como se mencionó anteriormente, para poder realizar todas las hazañas y destrezas en la barra, se necesita mostrar una mayor cantidad de piel de lo que se está acostumbrado normalmente, pues mientras más expuesta, mayor será nuestro desempeño en el deporte. El factor de mostrar la piel no es lo que provocaría en las estudiantes una mayor alza de su autoestima, sino que más bien, el hecho de poder utilizar este aspecto físico para las sesiones es lo que conllevaría a las participantes a contemplar su cuerpo y apreciar lo que este es capaz de realizar, ejerciendo directamente una relación con lo que pueden desempeñar en otras áreas no deportivas, específicamente en el área académica. A raíz de lo expresado anteriormente, se aprecian algunos cambios evidenciados en las estudiantes de la Universidad Austral de Chile que practican Pole Dance de manera voluntaria con respecto a su autoimagen, aspectos revelados en siguiente apartado.

\section{Método de investigación}

El enfoque utilizado en esta investigación es cualitativo exploratorio y el paradigma es interpretativo, donde es preciso que el autor "conozca el tema a partir de sus propios conocimientos para realizar una búsqueda exhaustiva que sea de ayuda para el estudio" (Martínez, 2013, p. 4). Las vivencias declaradas por las participantes arrojan conclusiones derivadas de la aplicación, y las respuestas aluden al proceso vivenciado. Debido a que este estudio se sumerge en investigaciones no tan exploradas y secciones poco indagadas, permite que, al realizar cualquier tipo de análisis bajo el mismo alero, se evidencien aspectos que sean de ayuda para futuras investigaciones. Cualquier descubrimiento que pueda resultar de una investigación exploratoria en relación al Pole Dance, será de importancia para la sección estudiada.

El muestreo fue por conveniencia, es decir, las participantes fueron seleccionadas por la investigadora, mientras que los criterios de selección se determinaron siempre que pertenecieran a estudiantes de la Universidad Austral de Chile que practicaran Pole Dance, tuvieran al menos dos meses de práctica de la disciplina, mantuvieran una asistencia regular a las sesiones, y que al momento de iniciar el estudio hubieran entregado el consentimiento informado. De este modo, el total de participantes se reduce a diez personas, quienes acceden al deporte pagando una mensualidad o ingresando desde los talleres gratuitos de la universidad.

Los instrumentos de recolección de información utilizados son encuesta de tipo Likert, grupo de discusión y entrevista semiestructurada. Con la encuesta tipo Likert se pudo añadir una mayor cantidad de datos específicos. Es el primer instrumento implementado en las participantes, donde a cada una se le entregó una encuesta a responder en un período de seis días. Con estos datos, se eligieron cuatro participantes para el grupo de discusión. El instrumento permitió mayor cantidad de respuestas y profundidad en el discurso, donde la metodología colectiva favoreció un ambiente de respuestas honestas. Finalmente, se realizaron entrevistas semiestructuradas a dos de las participantes del grupo de discusión que propiciaron declaraciones novedosas frente a sus emociones durante la práctica del deporte. Cada entrevista se realizó por separado, donde se respondieron a las preguntas de forma libre.

Los criterios éticos y de calidad seleccionados fueron en primer lugar la credibilidad, donde fue necesario que las participantes de la investigación reconocieran sus dichos como reales; en segundo lugar, la validez como criterio de rigor y orden metodológico estimado, evitando omitir pasos que desestimaran la autenticidad del estudio. En tercer lugar, estuvo la confidencialidad, volviéndose un factor obligatorio para las declaraciones de las participantes, y por último se entregaron formularios de consentimiento informado para ser completados por las participantes del grupo de estudio.

\section{Resultados}

Para analizar la encuesta tipo Likert, se dividió cada ítem de la encuesta en tres partes, donde se desarrollaron las explicaciones para su significado y posterior reflexión. El primer ítem (confianza en sí misma) poseía como puntaje máximo 25 puntos, equivalente a responder "totalmente de acuerdo" en todos los criterios de evaluación. Las afirmaciones del ítem se definen a continuación:

1) Desde que practico Pole Dance siento más seguridad con respecto a mis acciones.

2) Gracias al Pole Dance creo que soy capaz de lograr mis metas personales.

3) En Pole Dance siento libertad de mostrar mi cuerpo.

4) Desde que practico Pole Dance puedo expresarme tal cual soy.

5) Desde que practico Pole Dance me siento más fuerte mentalmente. 


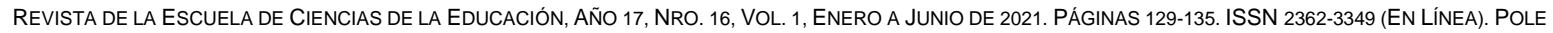
DANCE EN ESTUDIANTES DE LA UNIVERSIDAD AUSTRAL DE CHILE Y SU RELACIÓN CON LA AUTOIMÁGEN: REPERCUSIONES EN LA EDUCACIÓN SUPERIOR. IGNACIA CAROLINA OSORIO NAVARRO. LORETO DANIELA LIBUY CASTRO.

El puntaje mínimo obtenido en esta categoría fue de 19 puntos, mientras que una persona obtuvo el puntaje máximo. La mayoría de las encuestadas coincidían con estar entre los rangos de "totalmente de acuerdo" y "de acuerdo" en la totalidad de las preguntas. En el segundo ítem (convivencia) se observan los puntajes obtenidos por las estudiantes, que poseía un puntaje máximo de 20 puntos. Las afirmaciones de este ítem se definen a continuación:

1) Desde que practico Pole Dance tengo más capacidad de sociabilizar.

2) Actualmente, tengo mayor comunicación con mis compañeras de Pole Dance que cuando me inscribí.

3) El Pole Dance me ayuda a decir lo que pienso frente a otras personas.

4) Desde que estoy en Pole Dance he notado un cambio en mi vida social universitaria (positiva o negativa).

El puntaje mínimo obtenido en esta categoría fue de 14 puntos, mientras que una persona obtuvo como puntaje máximo 19 puntos. La mayoría de las encuestadas demuestran apuntar los aspectos de sociabilización mencionados en el instrumento, entre los niveles "totalmente de acuerdo" y "medianamente de acuerdo". En el tercer ítem (Pole Dance y responsabilidad académica) se observan los puntajes obtenidos por las estudiantes, el cual poseía como puntaje máximo 15 puntos. Las afirmaciones del ítem se definen a continuación:

1) Mediante el Pole Dance, ha mejorado mi actitud para cumplir con obligaciones universitarias.

2) Desde que estoy en Pole Dance siento mayor motivación para poder ejercer lo que estoy estudiando y formarme actualmente.

3) Desde que estoy en Pole Dance siento mayor compromiso con mis desafíos académicos y profesionales.

El puntaje mínimo obtenido en esta categoría fue de 10 puntos, mientras que una persona obtuvo el puntaje máximo.

El grupo de discusión y la entrevista semiestructurada se analizaron de manera conjunta. Se encuentran de este modo dos tipos de categorías que permitieron clasificar la información: la "categoría deductiva", donde la investigadora define conceptos en base a lo descrito y analizado anteriormente, sustentándose en los objetivos del estudio y en el marco teórico, por ende, los términos son directos y lo suficientemente amplios para generar sub-grupos que se definen como "categorías inductivas", que van creándose a medida que avanza el análisis de instrumentos. La siguiente tabla contiene dos columnas que integran ambas categorías.

\begin{tabular}{ll}
\hline \multicolumn{1}{c}{ Categorías Deductivas } & \multicolumn{1}{c}{ Categorías Inductivas } \\
\hline Macro Categoría A: & a.1 Sentidos y comodidad \\
Motivación hacia la práctica del Pole Dance & a.2 Sensualidad y autoestima \\
& b.1 Aprendizaje voluntario \\
Macro Categoría B: & b.2 Transformación interna \\
Pole Dance y Autoimagen & b.3 Comunidad de soporte \\
& b.4 Visión de cuerpo \\
Macro Categoría C: & c.1 Eficiencia académica \\
Pole Dance y su Relación Universitaria & c.2 Relación con externos al deporte
\end{tabular}

Tabla 1. Categorías de análisis. Elaboración propia.

La nomenclatura numérica utilizada para citar los instrumentos cualitativos de donde se extrajo la información fue "P1", correspondiente a documento primario de grupo de discusión; "P2" y "P3", como documentos primarios de entrevistas, y "(Parr X)", correspondiente al número de párrafo al que pertenece la cita inserta en el documento primario.

En la Macro Categoría A, se rescata una declaración situada en "sensualidad y autoestima", pues una de las participantes del grupo de discusión asegura que: 
ReVISTA DE LA ESCUELA dE CIENCIAS DE LA EdUCACIÓN, AÑO 17, NRO. 16, VOL. 1, ENERO A JUNIO DE 2021. PÁGINAS 129-135. ISSN 2362-3349 (EN LÍNEA). POLE DANCE EN ESTUDIANTES DE LA UNIVERSIDAD AUSTRAL DE CHILE Y SU RELACIÓN CON LA AUTOIMÁGEN: REPERCUSIONES EN LA EDUCACIÓN SUPERIOR. IGNACIA CAROLINA OSORIO NAVARRO. LORETO DANIELA LIBUY CASTRO.

"Lo que yo considero como mi motivación fue el Pole Exotic, porque me gusta la sensación de la sensualidad y el ejercicio, el querer tu cuerpo y aprender a moverte con él y dejar de auto limitarte" P1: $\mathrm{O} / 3$ (Parr 3).

Ante lo anterior, se desprende que este tipo de categoría del Pole Dance, permite la expresión del cuerpo en otras circunstancias que no pertenezcan a la rama deportiva, es decir, posee la habilidad de aceptar el cuerpo humano desde su capacidad para realizar ejercicios y movimientos. A raíz de lo anterior, Safatle (2016) hace énfasis en que se debe estudiar el desarrollo muscular en profundidad, pero sigue siendo una excelente técnica corporal. Es a través de este deporte que se permite conocer las posibilidades del cuerpo humano en cuanto a lo que puede realizar, pues fomenta además los rasgos de sensualidad de sus practicantes, al mismo tiempo que realizan actividad física.

En la Macro Categoría B se encuentra la categoría inductiva de "transformación interna", donde se reflejan los cambios de las estudiantes desde su comienzo en el deporte hasta la actualidad, interiorizándose en aspectos personales y/o emocionales que éstas desarrollan durante las sesiones. Una participante del grupo de discusión menciona que:

Yo siento que sí o sí es una sensación positiva. A mí en Pole Dance se me olvida todo, es que estoy tan concentrada en que me salgan las cosas haciendo lo que me gusta, que no puedo no salir contenta de eso, aunque me haya caído o no lo haya logrado, creo que siempre es una motivación a seguir volviendo y que quizá la otra clase me pueda salir, entonces es muy positivo P1: O/3 (Parr 15).

Aquí se refleja el sentir que expresan las estudiantes de Pole Dance, exponiendo su evolución con respecto a la apreciación que poseen de las clases, y lo que logran en ellas. Barra (2019) menciona que dentro de las clases "las chicas se sienten bien por dentro, porque ellas nunca piensan que pueden tener talento 0 condiciones físicas. (...). Les ayuda físicamente, pero también en el tema de creer en ellas y en sus propias capacidades" (Barra, 2019, p. 1).

La categoría deductiva "visión de cuerpo", expresa cómo las practicantes del deporte se han aceptado físicamente de manera exponencial durante el transcurso de las sesiones. Frente a este ámbito, otra integrante del grupo de discusión menciona que:

Yo por lo menos he notado cambios en lo físico. De hecho, yo antes nunca me preocupaba mucho de cómo me veía, sí me acomplejaba, pero no hacía nada al respecto. Y ahora yo noto que estoy más musculosa y a mí me encantan los músculos, y eso hace que me vaya gustando cada vez más Pole, porque he entrenado para eso" P1: O/6 (Parr 18).

Se desprende que la actividad física realizada en el Pole Dance permite que los músculos se desarrollen a medida que se evoluciona dentro del mismo, lo que es un factor importante para que las participantes se sientan menos acomplejadas con su cuerpo y más interesadas en cómo estos pueden progresar gracias a esta disciplina. Frente a esta afirmación, Holland (2012) expresa que "el esfuerzo no se trata solo de los cuerpos, sino también de lo que aprendemos de los cuerpos y las vidas que llevan" (Holland, 2012, p. 37).

Con respecto a la Macro Categoría C, en la categoría inductiva "eficiencia académica", se evidencia una relación entre la práctica del Pole Dance y las potencialidades que demuestran las estudiantes de la Universidad Austral de Chile en el ámbito académico. Una participante del grupo de estudio afirma con respecto al Pole Dance que:

Me hace sentir más perseverante, porque el gusto de querer que te salgan los trucos hace que tú le estés poniendo, y eso lo puedes llevar a otros contextos. Por ejemplo, para mí la u no vale mucho, pero sí sé que el Pole hace algo con respecto a eso. Yo la verdad es que siempre me converso, y lo mismo que me digo en Pole me lo digo al estudiar, y eso surgió del deporte, el echarme ánimo para el estudio P1: O/6 (Parr 18).

De esta declaración, se desprende que la práctica del Pole Dance ha permitido una mayor preocupación por sus actividades académicas, pues se ha considerado esta disciplina como un impulsor para realizar dichas tareas, creando una motivación con respecto a los quehaceres estudiantiles para rendir en ambos aspectos, lo que se condice con lo mencionado por Barra (2019), quien dice que "todo deporte necesita de disciplina y talento, pero también y sobre todo necesita de gente responsable. El Pole Dance a sus participantes las ha alineado y les ha enseñado el tema de la disciplina para un objetivo puntual” (Barra, 2019, p.2). 
ReVISTA DE LA ESCUELA dE CIENCIAS DE LA EdUCACIÓN, AÑO 17, NRO. 16, VOL. 1, ENERO A JUNIO DE 2021. PÁGINAS 129-135. ISSN 2362-3349 (EN LÍNEA). POLE DANCE EN ESTUDIANTES DE LA UNIVERSIDAD AUSTRAL DE CHILE Y SU RELACIÓN CON LA AUTOIMÁGEN: REPERCUSIONES EN LA EDUCACIÓN SUPERIOR. IGNACIA CAROLINA OSORIO NAVARRO. LORETO DANIELA LIBUY CASTRO.

\section{Discusión}

Es evidenciable que existen variados factores que pueden influir en la autoimagen de las practicantes de Pole Dance, lo que permite un mayor desenvolvimiento y socialización en el ámbito universitario. En el primer instrumento de análisis (encuesta tipo Likert) se demuestra en el ítem de "confianza en sí misma", que gracias a este deporte las estudiantes se sienten más seguras de sí, sobre todo con respecto a desenvolverse cotidianamente en otros contextos. Además, se hace énfasis en la seguridad desarrollada sobre sus cuerpos, lo que se vincula a lo expresado en el grupo de discusión y las entrevistas realizadas, pues las participantes del segundo y tercer instrumento de recolección de datos expresan que gracias al Pole Dance se sienten libres para mostrar su cuerpo sin prejuicios con respecto a lo que se pueda emitir de ellos.

En el ítem "convivencia" de la encuesta, las participantes afirman presenciar un cambio en su forma de sociabilización, ya sea entre deportistas o en otros contextos como la universidad. Dentro del grupo de discusión, las integrantes también afirman que, gracias a la realización de un deporte que las vuelve más plenas, obtienen la confianza para relacionarse con las personas de su entorno, lo que se vincula directamente con los puntajes obtenidos en el ítem II la encuesta, que especifica estos mismos aspectos.

En el ítem "Pole Dance y responsabilidad académica" de la encuesta, el grupo de estudio hace énfasis a que gracias al Pole Dance han podido organizar o modificar su eficiencia académica, es decir, han logrado generar algún cambio que repercute de manera positiva en sus tareas universitarias, lo que se relaciona con lo expresado en el grupo de discusión y las entrevistas semiestructuradas, pues en éstas sus participantes demostraron, que el hecho de realizar esta actividad de manera voluntaria, las ha convertido en personas disciplinadas, que es un factor necesario para sumar logros dentro del deporte.

\section{Conclusiones}

Uno de los objetivos específicos destaca las transformaciones que las estudiantes han obtenido a través del Pole Dance dentro del ambiente social enmarcado en la universidad. Se evidencia a través de lo expresado por el grupo de estudio que aquellos cambios sociales son de aspecto positivo para las participantes. Además, se extrae del análisis que las personas del círculo cercano universitario de las estudiantes, permiten y buscan hacerse parte del proceso vivenciado, lo que repercute en una mayor comunicación y desenvolvimiento entre sus pares, que ya no sólo interesan a la deportista, sino que también a su ambiente social académico. Como consecuencia, las participantes logran generar mayor diálogo con su entorno, aumentando de este modo los niveles de comunicación más allá de la actividad deportiva. El segundo objetivo específico expresa la relacionalidad existente entre el Pole Dance y la responsabilidad académica, obteniendo como resultado una relación positiva, donde las estudiantes manifiestan un mayor desempeño respecto a la organización de su tiempo para estudiar, además de mayor motivación para cumplir con lo exigido en la universidad.

Finalmente, el objetivo general de estudio busca encontrar las repercusiones que el Pole Dance ha producido en la autoimagen de las estudiantes, y según los datos recolectados se evidencia un cambio positivo con respecto a la autoestima de las participantes, pues todas expresan que gracias a este deporte se han convertido en personas más seguras y con mayor capacidad para expresar sus opiniones y relacionarse con otras personas. Aquellos cambios interfieren, directa o indirectamente, en su autoimagen pues les permite reflexionar sobre cómo se ven frente a la sociedad.

Uno de los temores que poseían las participantes de la investigación al ingresar al Pole Dance era enseñar mayor cantidad de piel de la que estaban acostumbradas. Sin embargo, con el paso de las clases las participantes destacan que este miedo fue desapareciendo. A través de las clases y el aumento de su confianza, las estudiantes advierten que son capaces de continuar su aprendizaje, lo que las vuelve más seguras, resaltando este aspecto para introducir a la autoimagen de cada una. La comodidad de las participantes dentro de las sesiones es un factor importante para desarrollar su autoimagen, pues propicia un mayor avance gracias a la presencia de las demás compañeras.

A raíz de los análisis, se confirma que todos los objetivos de la investigación se logran resolver de manera positiva, es decir, el Pole Dance si genera repercusiones en la autoimagen de estudiantes de la Universidad Austral de Chile, lo que les permite mejorar relaciones sociales, académicas y eficiencia dentro del mismo centro educativo. Dentro de las amenazas de la investigación, se encuentra la baja existencia de bases teóricas en el área que permitiera construir un estado del arte o un marco teórico más acabado, asunto que se puede minimizar con la divulgación de este estudio. Dentro de las proyecciones, se destaca que, a través del desarrollo de esta tesis emerjan preguntas que no pudieron ser respondidas, puesto que no correspondían al objeto de estudio como, por ejemplo, el Pole Dance infantil ¿tiene las mismas repercusiones en la autoimagen?, o ¿qué pasa con los varones que practican esta disciplina? De esta manera, quedan abiertas otras posibilidades de investigación para esta área nueva área deportiva. 


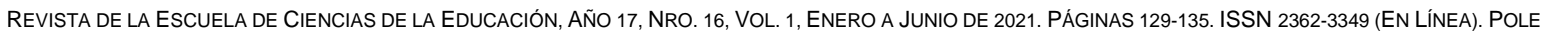
DANCE EN ESTUDIANTES DE LA UNIVERSIDAD AUSTRAL DE CHILE Y SU RELACIÓN CON LA AUTOIMÁGEN: REPERCUSIONES EN LA EDUCACIÓN SUPERIOR. IGNACIA CAROLINA OSORIO NAVARRO. LORETO DANIELA LIBUY CASTRO.

Referencias bibliográficas

Barra, Y. (2019). Entrevista semiestructurada [Manuscrito no publicado]. Chile: Instituto de ciencias de la educación, Universidad Austral de Chile.

Cifuentes, L. (2016). Autoimagen e inteligencia emocional, Estudio realizado con adolescentes entre 15 a 19 años de edad del Instituto Dr. Werner Ovalle, de la cabecera departamental de Quetzaltenango. [Tesis de grado, Universidad Rafael Landívar]. Recuperado en: http://recursosbiblio.url.edu.gt/tesiseortiz/2016/05/42/Cifuentes-Luz.pdf

Holland, S. (2012). Strippers and exotic dancers, Pole Dancing, empowerment and embodiment. Reino Unido: Palgrave.

Martínez, V. (2013). Paradigmas de investigación. Manual multimedia para el desarrollo de trabajos de investigación. Una visión desde la epistemología dialéctico crítica. Recuperado en: http://biblioteca.esucomex.cl/RCA/Paradigmas\%20de\%20investigaci\%C3\%B3n.pdf

Milena, S. (2014). El Pole Dance más allá de la sensualidad. El Heraldo. Recuperado en: https://www.elheraldo.co/tendencias/pole-dance-la-sensual-forma-de-hacer-ejercicio-155421

Morales, V. (2013). ¿Por qué es una buena elección el Pole Dance? En: Guía práctica del Pole Dance. Buenos Aires: Dunken.

Rodríguez, S. (2015). Los estudiantes universitarios de hoy: una visión multinivel. En: REDU: Revista de Docencia Universitaria, (13) 2, pp.91-124. Recuperado en: https://doi.org/10.4995/redu.2015.5440

Safatle (2016). Del Striptease al deporte: la reinvención del "Pole Dance". En: Infobae. https://www.infobae.com/salud/fitness/2016/08/06/del-striptease-al-deporte-la-reinvencion-del-pole-dance/

Torres, A. (2012). Características del estudiante universitario, Investigación y desarrollo social.

Valencia, C. (2019). "Qué es la autoimagen. La autoestima". Consultado el 19 de mayo de 2019. Recuperado en: https://www.laautoestima.com/autoimagen/ 